\title{
Extending the zero-derivative principle for slow-fast dynamical systems
}

\author{
Benoît, Eric; Brøns, Morten; Desroches, Mathieu; Krupa, Martin
}

\section{Published in:}

Zeitschrift fuer Angewandte Mathematik und Physik

Link to article, DOI:

$10.1007 / \mathrm{s} 00033-015-0552-8$

Publication date:

2015

Document Version

Peer reviewed version

Link back to DTU Orbit

Citation (APA):

Benoît, E., Brøns, M., Desroches, M., \& Krupa, M. (2015). Extending the zero-derivative principle for slow-fast dynamical systems. Zeitschrift fuer Angewandte Mathematik und Physik, 66(5), 2255-2270. https://doi.org/10.1007/s00033-015-0552-8

\section{General rights}

Copyright and moral rights for the publications made accessible in the public portal are retained by the authors and/or other copyright owners and it is a condition of accessing publications that users recognise and abide by the legal requirements associated with these rights.

- Users may download and print one copy of any publication from the public portal for the purpose of private study or research.

- You may not further distribute the material or use it for any profit-making activity or commercial gain

- You may freely distribute the URL identifying the publication in the public portal 


\title{
EXTENDING THE ZERO-DERIVATIVE PRINCIPLE FOR SLOW-FAST DYNAMICAL SYSTEMS
}

\author{
ERIC BENOÎT, MORTEN BRØNS, MATHIEU DESROCHES, AND MARTIN KRUPA
}

\begin{abstract}
Slow-fast systems often possess slow manifolds, that is invariant or locally invariant submanifolds on which the dynamics evolves on the slow time scale. For systems with explicit timescale separation the existence of slow manifolds is due to Fenichel theory and asymptotic expansions of such manifolds are easily obtained. In this paper we discuss methods of approximating slow manifolds using the so-called zero-derivative principle. We demonstrate several test functions that work for systems with explicit time scale separation including ones that can be generalized to systems without explicit timescale separation. We also discuss the possible spurious solutions, known as ghosts, as well as treat the Templator system as an example.
\end{abstract}

\section{INTRODUCTION}

Slow-fast systems naturally possess a very rich dynamics, which has been thoroughly investigated since the pioneering work of Van der Pol on relaxation oscillations [22]. Such systems provide deep mathematical problems and they also arise in many application areas, ranging from electronics to chemical kinetics and the study of biological rhythms.

The vast majority of models displaying slow-fast dynamics do not possess an explicit splitting between timescales. Such systems can be written in the general form

$$
\frac{\mathrm{d} z}{\mathrm{~d} t}=F(z, \varepsilon), \quad z \in \mathbb{R}^{N},
$$

where $0<\varepsilon \ll 1$ is a small parameter. In this paper, we wish to study slow-fast systems in the general form given by equation (1) assuming that, for small enough $\varepsilon$ and after some "manipulations" (change of time and/or variables), one can rewrite this system in the form of a slow-fast dynamical system with an explicit splitting of timescales, that is,

$$
\begin{aligned}
& \frac{\mathrm{d} x}{\mathrm{~d} t}=f(x, y, \varepsilon), \quad x \in \mathbb{R}^{n_{\mathrm{f}}}, \\
& \frac{\mathrm{d} y}{\mathrm{~d} t}=\varepsilon g(x, y, \varepsilon), \quad y \in \mathbb{R}^{n_{\mathrm{s}}},
\end{aligned}
$$

where $n_{\mathrm{f}}+n_{\mathrm{s}}=N$. Systems like (2) are usually referred to as singularly perturbed, meaning that the small parameter $\varepsilon$ multiplies the highest derivative of the differential equation (after rescaling time by a factor $\varepsilon$ in (2). This induces a clear splitting between fast variables $x$ and slow variables $y$. A good example of system initially written in the form of (1) and which can be put in the form of (2) after some rescalings is the so-called Templator model for self-replicating chemical dynamics [17]; see Section 5.

For "sufficiently small" $\varepsilon$, a fairly complete mathematical theory describes (locally) invariant manifolds associated with the flow of a singularly perturbed dynamical system of the form (2); it was derived through the work of Tikhonov [20] and Fenichel [7, 8], amongst others. In particular, the Fenichel theorems guarantee the existence and regularity of slow manifolds away from non-hyperbolic subsets of the unperturbed object, that is, the fast nullsurface $f(x, y, 0)=0$ defined for $\varepsilon=0$ and usually referred to as the critical manifold. Fenichel (slow) manifolds can then be approximated using various techniques, in particular via series expansions in powers of $\varepsilon$.

1991 Mathematics Subject Classification. 34D15 - 37D10 .

Key words and phrases. Slow-fast dynamics · Zero-derivative principle - Slow manifolds · Fenichel theory · Curvature · Intrinsic Low-dimensional Manifolds. Ghosts · Templator. 
Systems without an explicit timescale splitting are much more delicate to deal with at a general level; in a number of cases, computational approaches have been proposed in order to approximate slow manifolds, amongst which are the Intrinsic Low-Dimensional Manifold (ILDM) method [15], the Computational Singular Perturbation (CSP) technique [14], the iterative method of Fraser and Roussel [9, 19] and the zero-derivative principle [23]. These approaches supply algorithms which are intended to approximate slow manifolds for systems in the form of (1). On the other hand, there are rigorous mathematical results in the literature which show that if there is a way to go from the formulation (1) to the formulation (2), then the algorithms do indeed approximate slow manifolds [13, 24, 23]. In particular, in the case of the zero-derivative principle, estimates can be obtained in terms of approximations of the slow manifold to order $\varepsilon^{k}$ when looking at the zeros of the $k$-th derivative of the fast components of the original singularly perturbed system.

Another strategy, which applies to the case of one fast variable, is based on the curvature tensor associated with a given vector field. The idea was presented in the context of planar systems by Okuda at the end of the 1970s [16], Peng et al. [18] and then Brøns and Bar-Eli in the early 1990s [5]. More recently, it has been generalised to arbitrary dimension by Ginoux and collaborators $[11,12,10]$. It consists in computing the zeros of the $N \times N$ determinant

$$
G=\operatorname{det}\left(\begin{array}{llll}
\frac{\mathrm{d} z}{\mathrm{~d} t} & \frac{\mathrm{d}^{2} z}{\mathrm{~d} t^{2}} & \cdots & \frac{\mathrm{d}^{N} z}{\mathrm{~d} t^{N}}
\end{array}\right)
$$

which corresponds to the generalized curvature of the flow. Since the time-derivatives can be explicitly computed from the differential equations this provides a straightforward way of finding approximate slow manifolds analytically. In contrast, the ILDM and CSP methods require the computation of eigenvalues and eigenvectors for the Jacobian of $F$ which in general can be done only numerically. The curvature method works in the context of systems without explicit timescale separation, but it requires the evaluation of a very large determinant and produces many spurious solutions (known as ghosts, see below). Although slow manifolds are mapped to slow manifolds by coordinate changes the set $G=0$ is not mapped to the $G=0$ set in the new coordinates. This means it cannot be a slow manifold exactly. In this paper we establish that the approximation given by $G=0$ approximation is not any more accurate than the approximations derived using the zero derivative principle with generic test functions. From the practical point of view, using $G=0$ as a numerical approximation of a slow manifold incurs a very high cost of numerically or symbolically evaluating the flow derivatives of the vector field. The main motivation for the present work was to better understand how the curvature method works, with a more general goal in mind of deriving numerical methods for systems without an explicit timescale separation. Our conclusion is that the generalized curvature does indeed provide an example of a test function that works, but just one of many and not the simplest one.

In this paper we extend the results of [23] by applying the zero-derivative method to propose new test functions for slow manifolds (functions whose zero sets approximate the slow manifold) and, in particular, prove that $G$ is such a test function. Our idea is based on the observation that near regular points on the critical manifold the order of the approximation given by the slow components of the vector field can be well understood. This leads to an extended zero-derivative principle which gives a strategy to compute approximations of Fenichel manifolds in slow-fast systems without explicit timescale separation by simply considering the successive derivatives of any multi-linear combinations of all components of the vector fields. In particular, we show that our approach recovers the curvature method as an example of such a combination. The main advantage of this new approach is that it does not require any knowledge of the respective timescales present in the model. Furthermore, it is computationally much more efficient than having to compute a determinant. For a cleaner presentation we first consider the case $n_{\mathrm{f}}=1$, however we note here that most of our results can be generalized to the case of arbitrarily many fast dimensions. We carry out a generalization of some of the key results in Section 3.2.

Our approach shows, in particular, that estimates of slow manifolds can be obtained (at the cost of losing one order in $\varepsilon$ and having to satisfy a non-degeneracy condition $\partial_{x} g_{i} \neq 0$ ) when considering successive derivatives of a slow component of a singularly-perturbed vector field, away from non-hyperbolic regions. This non-degeneracy condition is not natural as it excludes systems with slowly-varying parameters (the 
evolution of the slow variables is independent of the fast variables), but in the context of chemical reaction models can be acceptable.

One draw-back of the time-derivative based methods is that apart from the solutions which are close to the slow manifold there are also other solutions which have no dynamical significance. In connection with the ILDM method such manifolds are known as ghosts $[2,3]$. We briefly discuss how these are associated with folds of the critical manifold where the assumptions for the Fenichel theory breaks down. Finally, we illustrate our method with a planar example of a slow-fast system without an explicit timescale separation, the so-called Templator model.

We conclude this Introduction by setting the stage for the rest of the paper. We consider the slow-fast system in dimension $n+l$

$$
\begin{array}{ll}
\frac{\mathrm{d} x}{\mathrm{~d} t}=f(x, y, \varepsilon), & x \in \mathbb{R}^{l}, \\
\frac{\mathrm{d} y}{\mathrm{~d} t}=\varepsilon g(x, y, \varepsilon), & y \in \mathbb{R}^{n} .
\end{array}
$$

We will assume that $f$ and $g$ are of class $C^{\infty}$, even if many of the results will hold even if the functions are only finitely many times continuous differentiable. We consider the dynamics near a point $\left(x_{0}, y_{0}\right)$ where

$$
f\left(x_{0}, y_{0}, 0\right)=0 \text { and } \partial_{x} f\left(x_{0}, y_{0}, 0\right) \text { is hyperbolic. }
$$

From this it follows that the critical manifold, defined by $f(x, y, 0)=0$, is the graph of a function $x=\phi_{0}(y)$ for $y$ in a neighborhood of $y_{0}$. Furthermore, it follows from Fenichel theory that for $r \geq 1$ there is a Fenichel manifold, a normally hyperbolic invariant $C^{r}$-manifold $W_{c}$ of dimension $n$ given by an equation $x=\phi(y, \varepsilon)$, defined for $\varepsilon$ small and $y$ in a neighborhood of $y_{0}$ such that

$$
\phi(y, \varepsilon)=\phi_{0}(y)+\mathcal{O}(\varepsilon) .
$$

We will fix $r$ in the following and it is to be understood that the derivatives we compute are defined up to order $r$ only.

The Fenichel manifold $W_{c}$ fulfills the following invariance equations:

$$
\begin{aligned}
\frac{\mathrm{d}}{\mathrm{d} t} \phi(y, \varepsilon) & =f(\phi(y, \varepsilon), y, \varepsilon), \\
\frac{\mathrm{d} y}{\mathrm{~d} t} & =\varepsilon g(\phi(y, \varepsilon), y, \varepsilon),
\end{aligned}
$$

or, by eliminating time,

$$
f(\phi(y, \varepsilon), y, \varepsilon)=\varepsilon \partial_{y} \phi(y, \varepsilon) g(\phi(y, \varepsilon), y, \varepsilon) .
$$

Unless explicitly mentioned, we will consider systems with one fast variable, $l=1$,

$$
\begin{array}{ll}
\frac{\mathrm{d} x}{\mathrm{~d} t}=f(x, y, \varepsilon), & x \in \mathbb{R}, \\
\frac{\mathrm{d} y}{\mathrm{~d} t}=\varepsilon g(x, y, \varepsilon), & y \in \mathbb{R}^{n} .
\end{array}
$$

In this case $f, \phi_{0}$ and $\phi$ are all scalar functions. The hyeprbolicity condition reduces to $\partial_{x} f\left(x_{0}, y_{0}, 0\right) \neq 0$ and the critical and the Fenichel manifold are hypersurfaces in the phase space.

\section{Estimates}

In this section we introduce some notation and state and prove three lemmas which are the key to prove the main theorems in the next section.

The $k$ th order time-derivative $\left(\mathrm{d}^{k} x / \mathrm{d} t^{k}, \mathrm{~d}^{k} y / \mathrm{d} t^{k}\right)$ of a solution to (9) can be computed from the system (9) as a function of $x, y$, and $\varepsilon$. We denote the $x$ and $y$ components $f^{(k)}$ and $\varepsilon g^{(k)}$ respectively and obtain 
the following recursion relations from the chain rule:

$$
\begin{array}{ll}
f^{(1)}=f, & f^{(k+1)}=\partial_{x} f^{(k)} f+\varepsilon \partial_{y} f^{(k)} g, \\
g^{(1)}=g, & g^{(k+1)}=\partial_{x} g^{(k)} f+\varepsilon \partial_{y} g^{(k)} g .
\end{array}
$$

With a change to the slow time $\tau=\varepsilon t$ the system (9) becomes

$$
\begin{aligned}
\varepsilon \frac{\mathrm{d} x}{\mathrm{~d} \tau} & =f(x, y, \varepsilon), \\
\frac{\mathrm{d} y}{\mathrm{~d} \tau} & =g(x, y, \varepsilon) .
\end{aligned}
$$

For $\varepsilon=0$ we get the slow subsystem

$$
\frac{\mathrm{d} y}{\mathrm{~d} \tau}=g\left(\phi_{0}(y), y, 0\right) \equiv h(y) .
$$

Again, we can find time derivatives $\mathrm{d}^{k} y / \mathrm{d} \tau^{k}$ of the solution $y$ (now as a function of the slow time $\tau$ ) recursively,

$$
h^{(1)}=h, \quad h^{(k+1)}=\partial_{y} h^{(k)} h .
$$

The following lemmas provide some fundamental estimates. We will write $\phi$ for $\phi(y, \varepsilon)$ and $\phi_{0}$ for $\phi_{0}(y)$ in the following.

Lemma 1. Let $W_{c}$ be a $C^{r}$ Fenichel manifold for the system (9) given by $x=\phi(y, \varepsilon)$. Let $k \leq r$ be a positive integer. Then

$$
\begin{aligned}
& f^{(k)}(\phi, y, \varepsilon)=\mathcal{O}\left(\varepsilon^{k}\right) \\
& g^{(k)}(\phi, y, \varepsilon)=h^{(k)}(y) \varepsilon^{k-1}+\mathcal{O}\left(\varepsilon^{k}\right)
\end{aligned}
$$

Proof. From the recursion relation (10a) and the invariance equation (8) we have

$$
\begin{aligned}
f^{(k+1)}(\phi, y, \varepsilon) & =\partial_{x} f^{(k)}(\phi, y, \varepsilon) f(\phi, y, \varepsilon)+\varepsilon \partial_{y} f^{(k)}(\phi, y, \varepsilon) g(\phi, y, \varepsilon) \\
& =\varepsilon\left(\partial_{x} f^{(k)}(\phi, y, \varepsilon) \partial_{y} \phi(y, \varepsilon)+\partial_{y} f^{(k)}(\phi, y, \varepsilon)\right) g(\phi, y, \varepsilon) \\
& =\varepsilon \partial_{y}\left(f^{(k)}(\phi, y, \varepsilon)\right) g(\phi, y, \varepsilon) .
\end{aligned}
$$

Define the $C^{r-1}$ function $\nu_{1}: \mathbb{R}^{n} \times \mathbb{R} \rightarrow \mathbb{R}$ by

$$
\nu_{1}(y, \varepsilon)=\partial_{y} \phi(y, \varepsilon) g(\phi, y, \varepsilon)
$$

such that $f(\phi, y, \varepsilon)=\varepsilon \nu_{1}(y, \varepsilon)$ from (8) and recursively, for $k \leq r$, the $\mathrm{C}^{r-k}$ function

$$
\nu_{k}(y, \varepsilon)=\partial_{y} \nu_{k-1}(y, \varepsilon) g(\phi, y, \varepsilon) .
$$

Then we have

$$
f^{(k)}(\phi, y, \varepsilon)=\varepsilon^{k} \nu_{k}(y, \varepsilon)
$$

which we now prove by induction. For $k=1$ the statement holds by construction. Assume now that (19) holds for some $k<r$. Then

$$
f^{(k+1)}(\phi, y, \varepsilon)=\varepsilon \partial_{y}\left(f^{(k)}(\phi, y, \varepsilon)\right) g(\phi, y, \varepsilon)=\varepsilon^{k+1} \partial_{y} \nu_{k}(y, \varepsilon) g(\phi, y, \varepsilon)=\varepsilon^{k+1} \nu_{k+1}(y, \varepsilon) .
$$

and the induction step is completed. The first estimate of the Lemma follows from (19). We proceed in a similar way for the second estimate. First we note that

$$
g^{(k+1)}(\phi, y, \varepsilon)=\varepsilon \partial_{y}\left(g^{(k)}(\phi, y, \varepsilon)\right) g(\phi, y, \varepsilon) .
$$

Define the $C^{r}$ function $\rho_{1}: \mathbb{R}^{n} \times \mathbb{R} \rightarrow \mathbb{R}^{n}$ by

$$
\rho_{1}(y, \varepsilon)=g(\phi, y, \varepsilon)
$$

and for $k \leq r+1$ the $C^{r-k+1}$ function $\rho_{k}: \mathbb{R}^{n} \times \mathbb{R} \rightarrow \mathbb{R}^{n}$ by

$$
\rho_{k}(y, \varepsilon)=\partial_{y} \rho_{k-1}(y, \varepsilon) g(\phi, y, \varepsilon) .
$$


Then as above we find by induction

$$
g^{(k)}(\phi, y, \varepsilon)=\varepsilon^{k-1} \rho_{k}(y, \varepsilon)
$$

for $k \leq r+1$. Furthermore,

$$
\rho_{k}(y, 0)=h^{(k)}(y) .
$$

Again, this is proved by induction: For $k=1$ the result is obvious. In the induction step, assume (25) holds for $k<r+1$. Then, using (13),

$$
\rho_{k+1}(y, 0)=\partial_{y} \rho_{k}(y, 0) g\left(\phi_{0}, y, 0\right)=\partial_{y} h^{(k)}(y) h(y)=h^{(k+1)}(y) .
$$

Combining (24) and (25) the second estimate of the Lemma follows.

Lemma 2. At the critical manifold the following equalities hold:

$$
\begin{aligned}
& \partial_{x} f^{(k)}\left(\phi_{0}, y, 0\right)=\partial_{x} f\left(\phi_{0}, y, 0\right)^{k}, \\
& \partial_{x} g^{(k)}\left(\phi_{0}, y, 0\right)=\partial_{x} g\left(\phi_{0}, y, 0\right) \partial_{x} f\left(\phi_{0}, y_{0}, 0\right)^{k-1} .
\end{aligned}
$$

Proof. Induction. We only prove the first identity. The proof of the second identity proceeds in the same way. The result obviously holds for $k=1$. Assume it holds for some $k$. Then using (10a) with $\varepsilon=0$ and $f\left(\phi_{0}, y, 0\right)=0$ yields

$$
\partial_{x} f^{(k+1)}\left(\phi_{0}, y, 0\right)=\partial_{x}\left(\partial_{x} f^{(k)} f\right)\left(\phi_{0}, y, 0\right)=\left(\partial_{x} f^{(k)} \partial_{x} f\right)\left(\phi_{0}, y, 0\right),
$$

and the result follows.

The proofs of Theorems 9 and 13 in the next sections rely on estimates of the functions $f^{(k)}$ and $g^{(k)}$ $\varepsilon^{m}$-close to the Fenichel manifold for various $k$ and $m$. We now establish these estimates. For $\xi \in \mathbb{R}$ we have from Taylor's theorem

$$
f^{(k)}\left(\phi+\varepsilon^{m} \xi, y, \varepsilon\right)=f^{(k)}(\phi, y, \varepsilon)+\varepsilon^{m} \partial_{x} f^{(k)}(\phi, y, \varepsilon) \xi+\mathcal{O}\left(\varepsilon^{2 m}\right)
$$

From Lemma 1 the first term on the right is $\mathcal{O}\left(\varepsilon^{k}\right)$. From (5) and $(27)$ it follows that $\partial_{x} f^{(k)}(\phi, y, \varepsilon) \neq 0$ for $y$ sufficiently close to $y_{0}$ such that the second term is $\mathcal{O}\left(\varepsilon^{m}\right)$. From similar arguments we find

$$
g^{(k)}\left(\phi+\varepsilon^{m} \xi, y, \varepsilon\right)=h^{(k)}(y) \varepsilon^{k-1}+\mathcal{O}\left(\varepsilon^{k}\right)+\varepsilon^{m} \partial_{x} g^{(k)}(\phi, y, \varepsilon) \xi+\mathcal{O}\left(\varepsilon^{2 m}\right) .
$$

This yields the following

\section{Lemma 3.}

$$
\begin{gathered}
f^{(k)}\left(\phi+\varepsilon^{m} \xi, y, \varepsilon\right)= \begin{cases}\mathcal{O}\left(\varepsilon^{k}\right) & \text { for } m \geq k \\
\partial_{x} f^{(k)}\left(\phi_{0}, y, 0\right) \xi \varepsilon^{m}+\mathcal{O}\left(\varepsilon^{m+1}\right) & \text { for } m<k\end{cases} \\
g^{(k)}\left(\phi+\varepsilon^{m} \xi, y, \varepsilon\right)= \begin{cases}h^{(k)}(y) \varepsilon^{k-1}+\mathcal{O}\left(\varepsilon^{k}\right) & \text { for } m>k-1 \\
\left(h^{(k)}(y)+\partial_{x} g^{(k)}\left(\phi_{0}, y, 0\right)\right) \varepsilon^{m}+\mathcal{O}\left(\varepsilon^{m+1}\right) & \text { for } m=k-1 \\
\partial_{x} g^{(k)}\left(\phi_{0}, y, 0\right) \xi \varepsilon^{m}+\mathcal{O}\left(\varepsilon^{m+1}\right) & \text { for } m<k-1\end{cases}
\end{gathered}
$$

\section{Approximately inVARiant hypersurfaces}

\subsection{Zero-derivative principles.}

Theorem 4. [23] Let $k>0$ be a positive integer. For $\varepsilon$ sufficiently small the equation $f^{(k)}(x, y, \varepsilon)=0$ restricted to a neighborhood of $\left(x_{0}, y_{0}, 0\right)$ defines a smooth function $x=\phi_{k}=\phi_{k}(y, \varepsilon)$. The function $\phi_{k}$ approximates the Fenichel manifold to order $k$,

$$
\phi_{k}-\phi=\mathcal{O}\left(\varepsilon^{k}\right) .
$$


Proof. We have $f^{(k)}\left(x_{0}, y_{0}, 0\right)=0$ according to Lemma 1 and $\partial_{x} f^{(k)}\left(x_{0}, y_{0}, 0\right)=\partial_{x} f\left(x_{0}, y_{0}, 0\right)^{k} \neq 0$ according to Lemma 2. It follows from the Implicit Function Theorem that there exists a function $x=\phi_{k}(y, \varepsilon)$, defined for $y$ close to $y_{0}$ and $\varepsilon$ close to 0 such that $\phi_{k}\left(y_{0}, 0\right)=x_{0}$ and $f^{(k)}\left(\phi_{k}(y, \varepsilon), y, \varepsilon\right)=0$. Furthermore, from the Mean Value Theorem,

$$
f^{(k)}\left(\phi_{k}, y, \varepsilon\right)-f^{(k)}(\phi, y, \varepsilon)=\partial_{x} f^{(k)}(\xi, y, \varepsilon)\left(\phi_{k}-\phi\right)
$$

for some $\xi$ between $\phi_{k}$ and $\phi$. The left hand side of $(35)$ is $\mathcal{O}\left(\varepsilon^{k}\right)$ according to Lemma 1 , and $\partial_{x} f^{(k)}(\xi, y, \varepsilon)$ is bounded away from 0 according to Lemma 2. From this (34) follows.

Theorem 5. Assume $\partial_{x} g_{i}\left(x_{0}, y_{0}, 0\right) \neq 0$. For $k \geq 2$ and $\varepsilon$ sufficiently small the equation $g_{i}^{(k)}(x, y, \varepsilon)=0$ restricted to a neighborhood of $\left(x_{0}, y_{0}\right)$ defines a smooth function $x=\psi_{k}=\psi_{k}(y, \varepsilon)$. The function $\psi_{k}$ approximates the Fenichel manifold to order $k-1$,

$$
\psi_{k}-\phi=\mathcal{O}\left(\varepsilon^{k-1}\right) .
$$

Proof. The proof proceeds as for Theorem 4, now using the identities (15) and (28). For completeness we retrace some of the steps. First note that, by Lemma $1, g_{i}^{(k)}\left(x_{0}, y_{0}, 0\right)=0$ for $k \geq 2$. Further, by Lemma $2, \partial_{x} g_{i}^{(k)}\left(x_{0}, y_{0}, 0\right) \neq 0$. It follows that there exists a unique $\psi_{k}(y, \varepsilon)$, solving $g_{i}^{(k)}=0$ and, by Lemma $\left.1, g_{i}^{(k)}(\phi(y), y)\right)=\mathcal{O}\left(\varepsilon^{k-1}\right)$. We now use the Mean Value Theorem argument as in the proof of Theorem 4 to conclude that estimate (36) holds.

Various combinations of higher order time derivatives can be used to obtain approximations to the Fenichel manifold. Here we consider one simple and useful example, the linear combination

$$
L_{k}=\alpha f^{(k)}+\sum_{i=1}^{n} \beta_{i} \varepsilon g^{(k)} .
$$

Note that $L_{k}$ can be easily evaluated for (1), this is due to our choice of including $\varepsilon$ in the components of the vector field corresponding to the slow directions. Hence this formula can easily be interpreted for a system without explicit timescale separation.

Theorem 6. Assume $\alpha \neq 0$ and let $\beta_{i}$ be arbitrary smooth functions of $x$ and $y$. For $\varepsilon$ sufficiently small the equation $L_{k}(x, y, \varepsilon)=0$ restricted to a neighborhood of $\left(x_{0}, y_{0}\right)$ defines a smooth function $x=\lambda_{k}(y, \varepsilon)$. The function $\lambda_{k}$ approximates the Fenichel manifold to order $k$,

$$
\lambda_{k}(y, \varepsilon)-\phi=\mathcal{O}\left(\varepsilon^{k}\right) .
$$

Proof. The proof proceeds as the previous ones. The existence of the function $\lambda_{k}$ is guaranteed from the Implicit Function Theorem as $\partial_{x} L\left(x_{0}, y_{0}, 0\right)=\alpha \partial_{x} f\left(x_{0}, y_{0}, 0\right)^{k} \neq 0$.

3.2. Arbitrarily many fast dimensions. The results of Section 3.1 can easily be generalized to arbitrarily many fast dimensions. Here we will show the generalization of Theorem 4 to the system (4). We leave the task of generalizing Theorems 5 and 6 to the reader.

Lemma 1 and Lemma 2 and their proofs carry over to the present setting. The functions $\nu_{k}$ introduced in the proof of Lemma 1 now map $\mathbb{R}^{n} \times \mathbb{R} \rightarrow \mathbb{R}^{l}$, and $\partial_{x} f\left(\phi_{0}, y_{0}, 0\right)^{k}$ in $(27)$ is the $k$-th power of the $l \times l$ matrix $\partial_{x} f\left(\phi_{0}, y_{0}, 0\right)$.

We will need the following higher-dimensional analogue of the Mean Value Theorem.

Lemma 7. There exists a constant $K>0$ such that

$$
\left\|f^{(k)}\left(\phi_{k}, y, \varepsilon\right)-f^{(k)}(\phi, y, \varepsilon)\right\| \geq K\left\|\phi_{k}-\phi\right\| .
$$


Proof. We carry out the elementary proof for completeness. We will use the continuity of $\partial_{x} f^{(k)}$ and the fact that $\phi-\phi_{0}$ as well as $\phi_{k}-\phi_{0}$ are $O(\varepsilon)$. We write

$$
\begin{aligned}
f^{(k)}(\phi, y, \varepsilon)-f^{(k)}\left(\phi_{k}, y, \varepsilon\right) & =\int_{0}^{1} \frac{d}{d s} f^{(k)}\left(\phi_{k}+s\left(\phi-\phi_{k}\right), y, \varepsilon\right) d s \\
& =\int_{0}^{1} \partial_{x} f^{(k)}\left(\phi_{k}+s\left(\phi-\phi_{k}\right), y, \varepsilon\right)\left(\phi-\phi_{k}\right) d s \\
& =\left(\partial_{x} f^{(k)}\left(x_{0}, y_{0}, 0\right)+O(\varepsilon)\right)\left(\phi-\phi_{k}\right) .
\end{aligned}
$$

The result now follows from the assumption that $\operatorname{det}\left(\partial_{x} f^{(k)}\left(\phi_{0}, y_{0}, 0\right)\right) \neq 0$.

We can now show the general zero derivative principle [23].

Theorem 8. Consider the $l+m$-dimensional system (4) and let $k>0$ be a positive integer. For $\varepsilon$ sufficiently small the equation $f^{(k)}(x, y, \varepsilon)=0$ restricted to a neighborhood of $\left(x_{0}, y_{0}, 0\right)$ defines a smooth vector function $x=\phi_{k}=\phi_{k}(y, \varepsilon)$. The function $\phi_{k}$ approximates the Fenichel manifold to order $k$,

$$
\phi_{k}-\phi=\mathcal{O}\left(\varepsilon^{k}\right) .
$$

Proof. The existence of $\phi_{k}$ follows from the Implicit Function Theorem as $f^{(k)}\left(x_{0}, y_{0}, 0\right)=0$ from Lemma 1 and the regularity of $\partial_{x} f^{(k)}\left(x_{0}, y_{0}, 0\right)$ from Lemma 2. According to Lemma 1

$$
f^{(k)}\left(\phi_{k}, y, \varepsilon\right)-f^{(k)}(\phi, y, \varepsilon)=\mathcal{O}\left(\varepsilon^{k}\right),
$$

and the estimate (41) now follows from Lemma 7.

3.3. The curvature determinant. In this section we return to the setting of one slow variable $(l=1)$ and consider the function $G$ defined by the $(n+1) \times(n+1)$ determinant

$$
G(x, y, \varepsilon)=\operatorname{det}\left(\begin{array}{cccc}
f^{(1)} & f^{(2)} & \cdots & f^{(n+1)} \\
\varepsilon g^{(1)} & \varepsilon g^{(2)} & \cdots & \varepsilon g^{(n+1)}
\end{array}\right) .
$$

Note that, similarly as in the case of $L_{k}, \varepsilon$ is included in the slow components of the vector field, which facilitates the use of this formula in the context of systems without explicit timescale separation. Recall the functions $h, h^{(1)}, h^{(2)}, \ldots$ defined by (12) and (13). We define the $n \times n$ determinant

$$
H(y)=\operatorname{det}\left(h^{(1)} \quad h^{(2)} \quad \cdots \quad h^{(n)}\right)
$$

Theorem 9. Assume $H\left(y_{0}\right) \neq 0$. For $\varepsilon$ sufficiently small the equation $G(x, y, \varepsilon)=0$ restricted to a neighborhood of $\left(x_{0}, y_{0}\right)$ defines a smooth function $x=\gamma(y, \varepsilon)$. The function $\gamma$ approximates the Fenichel manifold to order $n+1$,

$$
\gamma(y, \varepsilon)-\phi=\mathcal{O}\left(\varepsilon^{n+1}\right)
$$

Versions of Theorem 9 appear in the publications of Ginoux and collaborators $[11,12,10]$, however the hypothesis $H\left(y_{0}\right) \neq 0$ is not stated there. In Section 6 we show that the result fails if this hypothesis does not hold.

Proof. We first note as a direct consequence of Lemma 1 that

$$
G(\phi, y, \varepsilon)=\mathcal{O}\left(\varepsilon^{\frac{n(n+1)}{2}+1}\right) .
$$

We expand $G$ as follows

$$
G=\sum_{j=1}^{n+1} f^{(j)} G_{j},
$$

where $G_{j}$ is the $(1, j)$-cofactor for $G$. Using the estimates of Lemma 3 we find

$$
G_{j}\left(\phi+\varepsilon^{n} \xi, y, \varepsilon\right)=\mathcal{O}\left(\varepsilon^{\frac{n(n+1)}{2}-j+1}\right) .
$$


For $j=n+1$ the leading-order term is given by $H(y)$ such that we have the more precise estimate

$$
G_{n+1}\left(\phi+\varepsilon^{n} \xi, y, \varepsilon\right)=H(y) \varepsilon^{\frac{n(n+1)}{2}-n}+\mathcal{O}\left(\varepsilon^{\frac{n(n+1)}{2}-n+1}\right) .
$$

This yields for the terms in the sum (47)

$$
\left(f^{(j)} G_{j}\right)\left(\phi+\varepsilon^{n} \xi, y, \varepsilon\right)= \begin{cases}\mathcal{O}\left(\varepsilon^{\frac{n(n+1)}{2}+1}\right) & \text { for } j \leq n \\ H(y) \partial_{x} f^{(n+1)}(\phi, y, \varepsilon) \xi \varepsilon^{\frac{n(n+1)}{2}}+\mathcal{O}\left(\varepsilon^{\frac{n(n+1)}{2}+1}\right) & \text { for } j=n+1\end{cases}
$$

such that

$$
G\left(\phi+\varepsilon^{n} \xi, y, \varepsilon\right)=H(y) \partial_{x} f^{(n+1)}(\phi, y, \varepsilon) \xi \varepsilon^{\frac{n(n+1)}{2}}+\mathcal{O}\left(\varepsilon^{\frac{n(n+1)}{2}+1}\right) .
$$

The theorem now follows from the Implicit Function Theorem applied to the function $G / \varepsilon^{\frac{n(n+1)}{n}}$.

Remark 10. Note that hypothesis $H\left(y_{0}\right) \neq 0$ is essential and its failure can lead to the appearance of spurious solutions known as ghosts, see Section 6.

\section{The ILDM Method AND THE ZERO-DERIVATIVE PRINCIPLE}

A popular method for finding approximations of slow manifolds has been the ILDM (Intrinsic LowDimensional Manifold) method [15, 13]. In this section we discuss how to connect this method to the zeroderivative principle. We first review the construction of intrinsic low-dimensional manifolds (approximate slow manifolds). We consider the $N$-dimensional system

$$
\dot{z}=F(z), \quad z \in \mathbb{R}^{N} .
$$

For simplicity, we make the generic assumption that the eigenvalues $\lambda_{p}(z), p=1, \ldots, N$ of the Jacobian matrix $J(z)=D F(z)$ are all different, such that there is a basis of eigenvectors $v_{p}=v_{p}(z)$ for the tangent space to $\mathbb{R}^{N}$ at $z$. For complex eigenvalues we use the real and imaginary parts of an eigenvector as base vectors. Order the eigenvalues such that

$$
\operatorname{Re} \lambda_{1} \leq \operatorname{Re} \lambda_{2} \leq \cdots \leq \operatorname{Re} \lambda_{N-1} \leq \operatorname{Re} \lambda_{N}
$$

Equality occurs only for conjugate pairs of complex eigenvalues. It is assumed that there is a spectral gap at, say, $\lambda_{n_{\mathrm{f}}}$ such that $\operatorname{Re} \lambda_{n_{\mathrm{f}}} \ll \operatorname{Re} \lambda_{n_{\mathrm{f}}+1}$, clearly dividing the eigenvalues into fast ones and slow ones. The ILDM is then defined as the manifold of dimension $n_{s}=N-n_{\mathrm{f}}$ where the fast components of $F$ in the eigenvector base vanish. In formulas, let $V$ be the matrix where the eigenvectors are columns. Then $F$ can be expanded in the eigenvector basis as

$$
F=V \alpha
$$

where $\alpha=\left(\alpha_{1}, \alpha_{2}, \ldots, \alpha_{N}\right)^{T}$. This yields

$$
\alpha=V^{-1} F
$$

and the ILDM is then defined from

$$
\alpha_{1}=\cdots=\alpha_{n_{\mathrm{f}}}=0 .
$$

For a singular perturbation problem of the form (2), a spectral gap is present with $n_{\mathrm{f}}$ fast eigenvalues. For such systems Kaper and Kaper [13] show that the ILDM of dimension $n_{\mathrm{s}}$ agrees with the Fenichel manifold to order $\mathcal{O}\left(\varepsilon^{2}\right)$.

Define the $N \times\left(n_{\mathrm{s}}+1\right)$ matrix

$$
A=\left(\begin{array}{llll}
F & J F & \cdots & J^{n_{\mathrm{s}}} F
\end{array}\right) .
$$

Theorem 11. On the $I L D M$ defined by (56) the rank of $A$ is not maximal (i.e. $\leq n_{\mathrm{s}}$.)

Proof. The columns of $A$ are

$$
F=\sum_{p=1}^{N} \alpha_{p} v_{p}, \quad J F=\sum_{p=1}^{N} \alpha_{p} \lambda_{p} v_{p}, \quad \ldots \quad J^{n_{\mathrm{s}}} F=\sum_{p=1}^{N} \alpha_{p} \lambda_{p}^{n_{\mathrm{s}}} v_{p},
$$


such that $A_{n_{\mathrm{s}}}=V B$, where

$$
B=\left(\begin{array}{cccc}
\alpha_{1} & \alpha_{1} \lambda_{1} & \cdots & \alpha_{1} \lambda_{1}^{n_{\mathrm{s}}} \\
\alpha_{2} & \alpha_{2} \lambda_{2} & \cdots & \alpha_{2} \lambda_{2}^{n_{\mathrm{s}}} \\
\vdots & \vdots & \ddots & \vdots \\
\alpha_{N} & \alpha_{N} \lambda_{N} & \cdots & \alpha_{N} \lambda_{N}^{n_{\mathrm{s}}}
\end{array}\right)
$$

The rank of $B_{n_{\mathrm{s}}}$, which is equal to the rank of $A_{n_{\mathrm{s}}}$, is not maximal if and only if all minors of order $n_{\mathrm{s}}+1$ are zero. An $\left(n_{\mathrm{s}}+1\right) \times\left(n_{\mathrm{s}}+1\right)$ submatrix of $B$ can be factorized into a diagonal matrix with entries $\alpha_{i}, i \in I$, where $I$ is an index set with $n_{\mathrm{s}}+1$ elements, and a Vandermonde matrix with entries $\lambda_{i}^{j}, i \in I, j=0, \ldots n_{\mathrm{s}}$. Since the eigenvalues are assumed to be distinct, the determinant of the Vandermonde matrix is nonzero. Hence, the rank of $A$ is not maximal if and only if

$$
\prod_{i \in I} \alpha_{i}=0 \text { for all index sets } I \text { with } n_{\mathrm{s}}+1 \text { elements. }
$$

It is easy to see that (56) implies (60).

For the case of one fast variable, $n_{\mathrm{s}}=N-1$, the matrix $A$ is square of order $N$ and its rank is not maximal if and only if the determinant is zero. Defining the function

$$
M P=\operatorname{det}\left(\begin{array}{llll}
F & J F & \cdots & J^{N-1} F
\end{array}\right)
$$

we get from Theorem 11

Theorem 12. On an $N-1$ dimensional ILDM the function $M P$ vanishes.

The function $M P$ is explicitly computable by differentiation, while no analytical expression for the eigenvalues $\alpha_{i}$ defining the ILDM can be found in general. Hence, solving $M P=0$ to find an approximate slow manifold is a simple and attractive approach. However, the full solution set for $M P=0$ is larger than the ILDM, as also surfaces where slow components of $F$ vanish are included. These are known as ghosts and can be interpreted in the ILDM sense as occurring from identifying the fast eigen-directions incorrectly $[2,3]$. See also Section 6. Furthermore, we will now show that although a rigorous foundation for the ILDM method is already provided in [13], the zero-derivative can also be used to establish the soundness and accuracy of the method. For simplicity we assume that the system has only one fast variable and establish an analogue of Theorem 9. A generalization to arbitrarily many fast variables is conceptually straightforward, but requires more involved notation. To prove such a result we need a non-degeneracy condition similar to $H\left(y_{0}\right) \neq 0$, see (44) for the definition of $H$. We have

Theorem 13. Let $F$ be given as in (9) and assume the non-degeneracy condition (64). For $\varepsilon$ sufficiently small the equation $M P(x, y, \varepsilon)=0$ for the slow-fast system (9) restricted to a neighborhood of $\left(x_{0}, y_{0}\right)$ defines a smooth function $x=\mu=\mu(y, \varepsilon)$. The function $\mu$ approximates the Fenichel manifold to order 2 ,

$$
\mu-\phi=\mathcal{O}\left(\varepsilon^{2}\right) .
$$

Proof. We will take advantage of the fact that $M P$ can be written as a function of $F$ and $F^{(2)}=J F$, $M P=\left(\begin{array}{lllll}F & F^{(2)} & J F^{(2)} & \cdots & J^{N-2} F^{(2)}\end{array}\right)$, . Apart from the first column, each entry of $M P$ is a linear combination of $f^{(2)}$ and $\varepsilon g_{i}^{(2)}, i=1, \ldots, n$. Hence $M P$ is a homogeneous polynomial of degree $n$ in the variables $f^{(2)}$ and $\varepsilon g_{i}^{(2)}$,

$$
M P=\sum_{p+|q|=n} A_{p, q}(x, y, \varepsilon) f^{(2)}(x, y, \varepsilon)^{p}\left(\varepsilon g^{(2)}(x, y, \varepsilon)\right)^{q},
$$

where $q=\left(q_{1}, \ldots, q_{n}\right)$ is a multi-index. As in Theorem 9 , we consider the equation $M P(\phi+\varepsilon \xi, y, \varepsilon)=0$ and find that it can be solved for $\xi$ when $\varepsilon$ is sufficiently small using the Implicit Function Theorem assuming

$$
\sum_{|q|=n-1} A_{1, q}\left(x_{0}, y_{0}, 0\right) \partial_{x} f^{(2)}\left(x_{0}, y_{0}, 0\right) h^{(2)}\left(y_{0}\right)^{q} \neq 0
$$


We leave the details to the reader.

Clearly, the approach behind the proofs of Theorems 9 and 13 can be used for a large class of functions of the time-derivatives $f^{(k)}$ and $\varepsilon g^{(k)}$. It is worth noting that these functions do not need to have any simple interpretation in terms of curvature and other differential geometric properties of the solution curves.

\section{Applichtion to the Templator model}

Here we apply the theory to the Templator model for the kinetics of a self-replicating chemical system. The system consists of two species $X$ and $T$, where $T$ acts as a template for its own production. See $[17,21,1]$ for further details of the model and its biological significance. The equations are

$$
\begin{aligned}
& \frac{\mathrm{d} X}{\mathrm{~d} t}=r-k_{u} X^{2}-k_{T} X^{2} T \\
& \frac{\mathrm{d} T}{\mathrm{~d} t}=k_{u} X^{2}+k_{T} X^{2} T-\frac{q T}{K+T} .
\end{aligned}
$$

As in previous studies we consider $k_{u}=0.01, k_{T}=1, K=0.02, q=1$. Typically $r$ is considered a bifurcation parameter which we here fix to 0.9 .

The system exhibits a slow-fast behavior, but there is no obvious small parameter. However, in an analysis of canard explosions in the system Brøns [4] found two different transformations which identified the slow-fast structure in different regions of the phase plane under the main assumption that $K$ is small.

Letting $K=\varepsilon$ and assuming further that $r$ and $q$ are close such that $r=q+c \varepsilon$ the system can be rewritten

In the limit $\varepsilon=0$ the curve

$$
\begin{aligned}
& \frac{\mathrm{d} X}{\mathrm{~d} t}=q-k_{u} X^{2}-k_{T} X^{2} T+c \varepsilon \\
& \frac{\mathrm{d} T}{\mathrm{~d} t}=-\left(q-k_{u} X^{2}-k_{T} X^{2} T\right)-\frac{\varepsilon q}{\varepsilon+T} .
\end{aligned}
$$

consists of equilibria and hence is a critical manifold. The system can be brought on standard form (9) by the transformation $x=T-X, y=T+X$ such that $x$ is a fast variable and $y$ is slow,

$$
\begin{aligned}
& \frac{\mathrm{d} x}{\mathrm{~d} t}=2\left(q-k_{u} X^{2}+k_{T} X^{2} T\right)-\varepsilon\left(\frac{q}{c+\varepsilon+T}\right), \\
& \frac{\mathrm{d} y}{\mathrm{~d} t}=\varepsilon\left(c-\frac{q}{\varepsilon+T}\right) .
\end{aligned}
$$

If $T$ is small, the last term in (66b) is not uniformly bounded as $\varepsilon \rightarrow 0$ and another scaling must be used. Assuming that $k_{u}$ is also small we now let $K=\varepsilon^{2 / 3}$ and further defining $T=\varepsilon^{2 / 3} u, X=$ $\varepsilon^{-1 / 3} v, k_{u}=\varepsilon^{2 / 3} \alpha, t=\varepsilon^{2 / 3} \tau$ we get

$$
\begin{aligned}
& \frac{\mathrm{d} u}{\mathrm{~d} \tau}=\alpha v^{2}+k_{T} u v^{2}-\frac{q u}{1+u}, \\
& \frac{\mathrm{d} v}{\mathrm{~d} \tau}=\varepsilon\left(r-\alpha v^{2}-k_{T} u v^{2}\right),
\end{aligned}
$$

which again is in the form (9), and where the critical manifold $\mathrm{d} u / \mathrm{d} \tau=0$ in original variables is given by

$$
k_{u} X+k_{T} X^{2} T-\frac{q T}{K+T}=0 .
$$

For the present choice of parameters the system has an attracting limit cycle. See Figure 1. We see that for high values of $T$ the limit cycle closely follows the critical manifold for (68) as expected. The Fenichel theorem breaks down when the critical manifold has a fold in the $x, y$ coordinate system, corresponding to a point where its slope is equal to -1 in the original $X, T$ variables. Close to this point $F_{1}$ the trajectory leaves the critical manifold and goes to an attracting branch of the critical manifold for the system (69). 


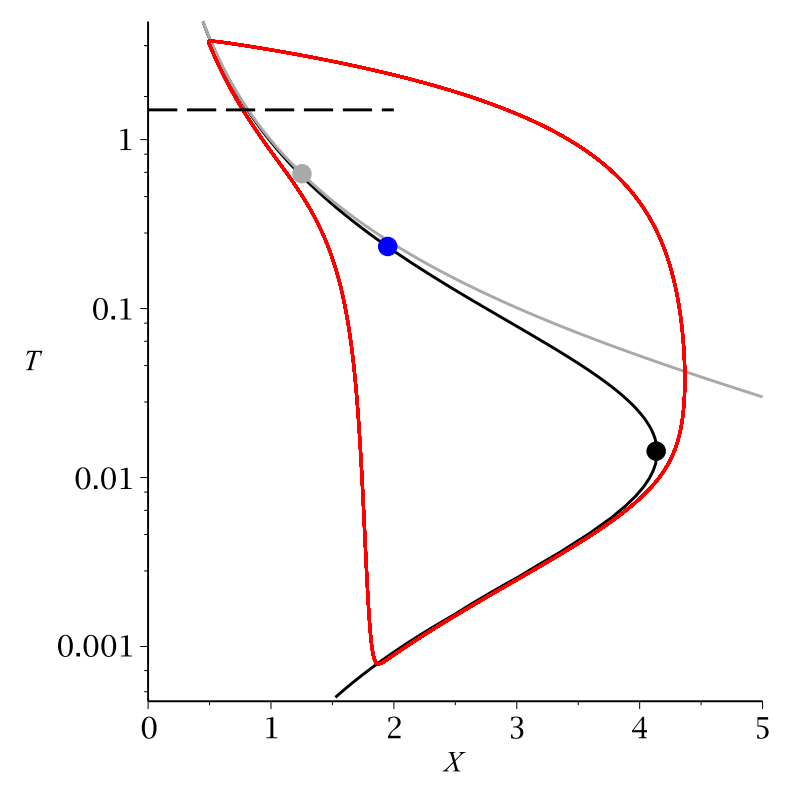

Figure 1. Red curve: Limit cycle for the Templator (65). Gray curve: Critical manifold for the transformed system (68). The gray point is the fold point $F_{1}$. Black curve: Critical manifold for the transformed system (69). The black point is the fold point $F_{2}$. The blue point is the unstable equilibrium. The dashed line shows where a Fenichel manifold and the approximations are compared in Figure 3. Note the logarithmic scale on the $T$-axis in this and the following figures.

Here it stays until the fold point $F_{2}$ of this manifold is reached. Since the slow variable for (69) is proportional to $X$ and the fast variable is proportional to $T$ this occurs where the Fenichel manifold has a vertical tangent in the $X, T$-space. After leaving the critical manifold the trajectory rapidly returns to the other critical manifold at high $T$.

Figure 2 show various solution sets which are obtained using the zero-derivative principle for both variables $X$ and $T$ as well as the zero set for $G$. Interestingly, we see that the manifolds have branches close to the hyperbolic parts of the critical manifolds for both scalings (66) and (69) and hence the zero-derivative principle effectively captures the dynamics independently of the identification of a small parameter. However, the global solution sets are very complicated and have branches which do not approximate any slow manifold. These branches are the ghosts. Close to the fold points of the critical manifolds the branches which approximate a Fenichel manifold merge with a ghost in a fold. Furthermore, we see that the solution to $G=0$ has a double point at the equilibrium where a ghost intersects the approximation to the Fenichel manifold transversally.

A more quantitative demonstration of the accuracy of the methods is shown in Figure 3: A Fenichel manifold close to the critical manifold (67) is obtained by numerical integration from an initial point with a high value of $T$ and a low value of $X$. The solution is continued until $T=1.5$ is reached, where $X=X_{s}$. For the various manifolds we compute the value $X=X_{m}$ at $T=1.5$ and show the relative error $\left|X_{m}-X_{s}\right| / X_{m}$ as a function of the order of the approximation. Note that we choose to use the relative error as it gives information about how many digits are correct, which is not the case with the absolute error. In addition to the simple quantities $X^{(k)}$ and $T^{(k)}$ we also show the linear combinations $X^{(k)}-T^{(k)}$ and $X^{(k)}+T^{(k)}$. In general the approximation improves as a function of the order, and the methods give practically the same results for $k=5$. An exception is $X^{(k)}+T^{(k)}$ which lags behind the other methods. This is to be expected, since the variable $X+T$ is a slow variable in (68). The observed linear relation between $k$ and the logarithmic error is to be expected from the theory. Also the result for $G$ compares well with the other methods at $k=2$ as expected. 
(a)

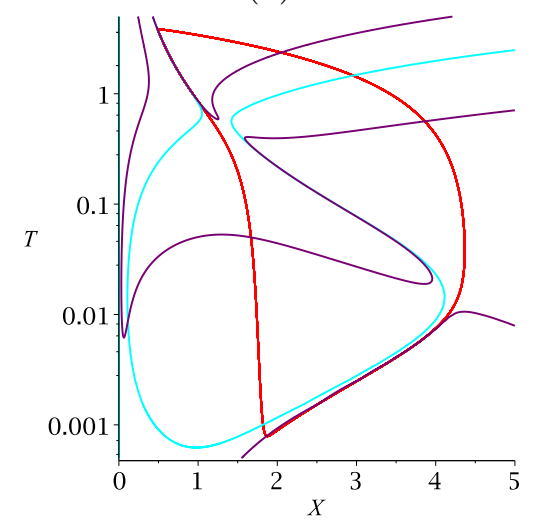

(d)

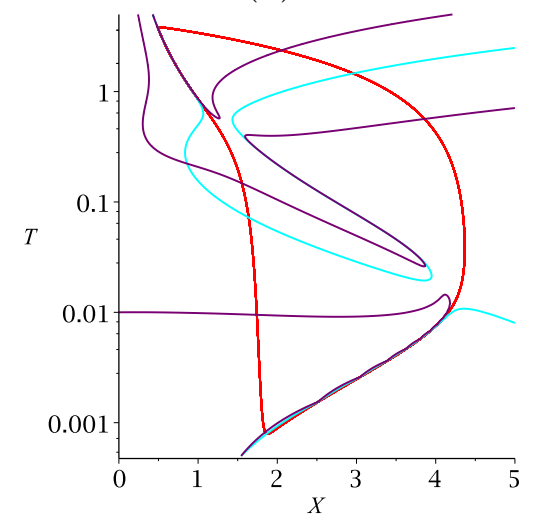

(g)

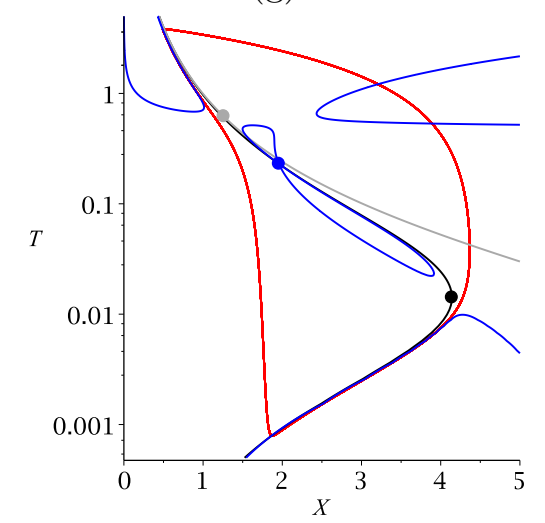

(b)

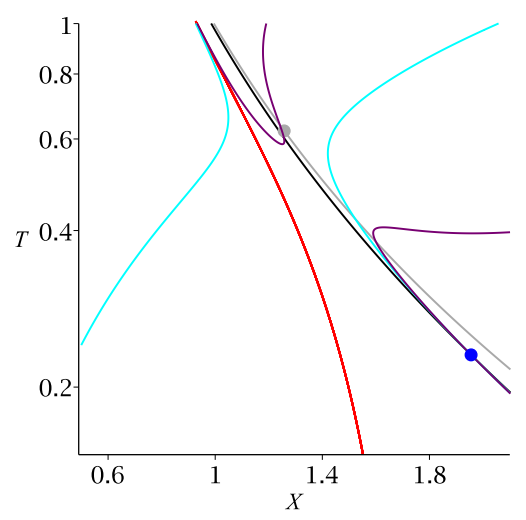

(e)

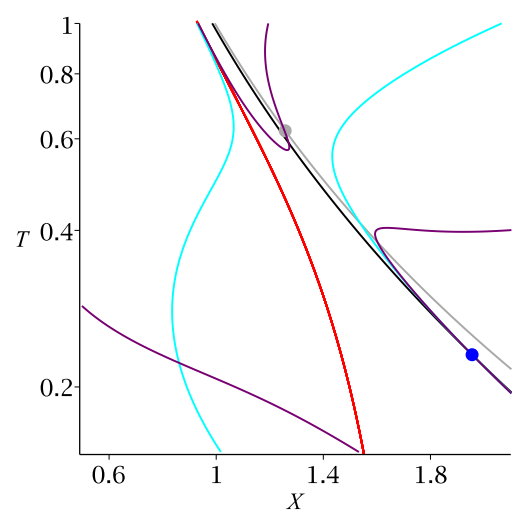

(h)

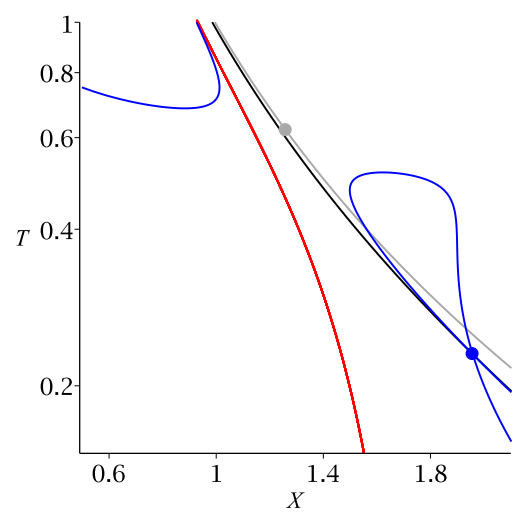

(c)

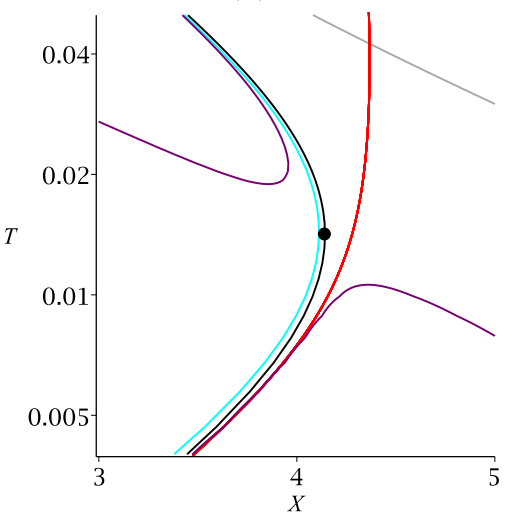

(f)

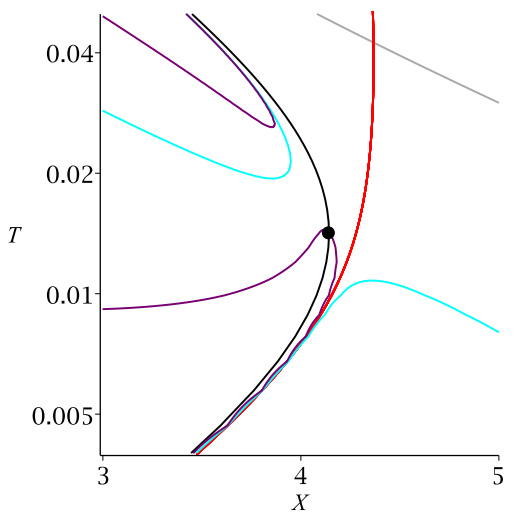

(i)

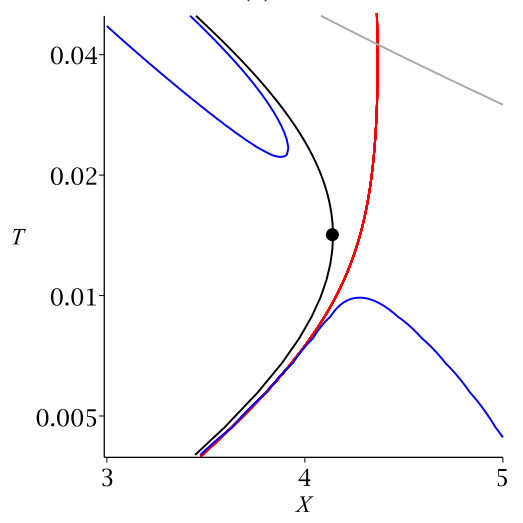

Figure 2. (a) Solutions to $X^{(2)}=0$ (cyan curve) and $X^{(3)}=0$ (purple curve) for the Templator. The red curve is the limit cycle. (b) and (c) are close-ups near $F_{1}$ and $F_{2}$ respectively. The critical manifolds are also shown here. Panels (d)-(f) show $T^{(2)}=0$ (cyan curve) and $T^{(3)}=0$ (purple curve). Panels (g)-(i) show the solution to $G=0$ (blue curve).

\section{Ghosts}

In the previous section we demonstrated numerically that the global structure of the zero set for various combinations of time derivatives can be complicated and contain ghost branches which do not approximate slow manifolds. Here we give a more general analysis of the structure of these sets for the case $n=1$. 


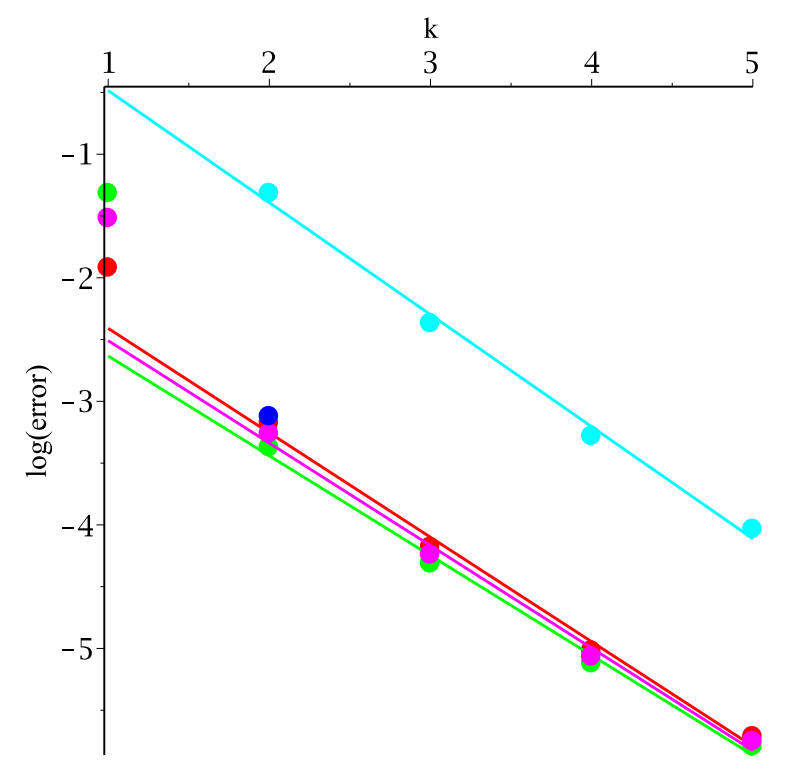

Figure 3. Relative deviations for various approximations to the Fenichel manifold at $T=$ 1.5. Red: $X^{(k)}=0$. Green: $T^{(k)}=0$. Magenta: $X^{(k)}-T^{(k)}=0$. Cyan: $X^{(k)}+T^{(k)}=0$. The single blue marker shows $G=0$. The lines are linear fits based on the points with $k \geq 2$.

Consider first the equation $f^{(k)}(x, y, \varepsilon)=0$. From Eqn. (10a) we have for $\varepsilon=0$

$$
f^{(k)}(x, y, 0)=\partial_{x} f^{(k-1)}(x, y, 0) f(x, y, 0)=\partial_{x} f(x, y, 0)^{k-1} f(x, y, 0)
$$

such that the solution to $f^{(k)}=0$ is the union of the solutions to $f=0$ and $\partial_{x} f=0$. For $\varepsilon$ not zero the first solution perturbs into an approximation to a Fenichel manifold while the latter perturbs into a ghost. For $\varepsilon=0$ the two solution sets have a common point at a fold of the critical manifold where $f=\partial_{x} f=0$. Generically the two solution branches going through this double point unfolds into two separate branches for $\varepsilon$ not zero, each consisting of an approximate Fenichel manifold which meets a ghost in a fold point. This local structure is clearly visible in Figure 2(f). Close to the fold point $F_{2}, T$ is a fast variable such that the curves shown are $f^{(2)}=0$ and $f^{(3)}=0$.

For the equation $g^{(k)}(x, y, \varepsilon)=0$ we have at $\varepsilon=0$

$$
g^{(k)}(x, y, 0)=\partial_{x} g^{(k-1)}(x, y, 0) f(x, y, 0)=\partial_{x} g(x, y, 0) \partial_{x} f(x, y, 0)^{k-2} f(x, y, 0) .
$$

Now the zero set is composed of three components, the solutions to $f=0, \partial_{x} f=0$, and $\partial_{x} g=0$. Again the first perturbs into an approximation of a Fenichel manifold for $\varepsilon$ not zero, while the latter produce ghosts. Thus, a more complex ghost structure results when using $g^{(k)}$ rather than $f^{(k)}$.

For $G=0$ we let

$$
\tilde{G}=\frac{G}{\varepsilon}=f^{(1)} g^{(2)}-f^{(2)} g^{(1)}
$$

Note that condition $H(y) \neq 0$ for $N=1$ reduces to $g(\phi(y), y), 0) \neq 0$. Hence, if $g(\phi(y), y), 0)=0$, which corresponds to an equilibrium point the assumptions of Theorem 9 are violated. This case was considered by [6], who proved that the solutions set of $G=0$ has a transcritical point near this singularity. Such a transcritical point is shown for the templator model in Figure $2(\mathrm{~g})$. We expect that in higher codimensions the failure of the condition $H=0$ leads to similar, but much more complicated degeneracies, in particular approximations of the slow manifolds of lower order in $\varepsilon$ may exist in the vicinity of such points. A similar condition holds in the context of ILDM with respect to the suitable non-degeneracy condition, see Theorem 13 . 


\section{Discussion}

In this paper we extend the zero-derivative principle for slow-fast dynamical systems. Our main contribution is to provide a much larger class of test functions to approximate slow manifolds and to give precise estimates on the resulting approximation. In particular, we show that considering the slow components of the vector fields works almost equally well as with the fast components (which is the classical zero-derivative principle), at the expense of one order of approximation in the small parameter.

In light of our approximation results, we are able to revisit curvature-based methods and show that they correspond to a special choice of the test function to approximate slow manifolds. In particular, we show that the choice of determinants is far from optimal, in terms of computational efficiency and number of ghosts.

A further study of ghost manifolds in such problems is ongoing work. Indeed, ghosts appear when several solution branches to the problem meet up, in link with the failure of some of the non-degeneracy conditions (which are problem-dependent). Our goal in this direction is to do a more systematic analysis of ghosts, their creation and number, using singularity theory.

Finally, we plan to apply our results to more complicated examples where there is no explicit (or uniform) timescale separation, in particular in the context of conductance-based neuronal models.

\section{REFERENCES}

[1] K. M. Beutel and E. Peacock-López. Complex dynamics in a cross-catalytic self-replication mechanism. The Journal of Chemical Physics, 126(12): 125104, 2007.

[2] S. Borok, I. Goldfarb, and V. Gol'dshtein. About non-coincidence of invariant manifolds and intrinsic low dimensional manifolds (ILDM). Communications in Nonlinear Science and Numerical Simulation, 13: 1029-1038, 2008.

[3] S. Borok, I. Goldfarb, and V. Gol'dshtein. Causes for "ghost" manifolds. Communications in Nonlinear Science and Numerical Simulation, 14: 1791-1795, 2009.

[4] M. Brøns. Canard explosion of limit cycles in templator models of self-replication mechanisms. The Journal of Chemical Physics, 134(14): 144105, 2011.

[5] M. Brøns and K. Bar-Eli. Asymptotic analysis of canards in the EOE equations and the role of the inflection line. Proceedings of the Royal Society of London. Series A: Mathematical, Physical and Engineering Sciences, 445(1924): 305-322, 1994.

[6] M. Desroches, M. Krupa and S. Rodrigues. Inflection, canards and excitability threshold in neuronal models. Journal of Mathematical Biology, 67(4): 989-1017, 2013.

[7] N. Fenichel. Persistence and smoothness of invariant manifolds for flows. Indiana University Mathematics Journal, 21(3): 193-226, 1971.

[8] N. Fenichel. Geometric singular perturbation theory for ordinary differential equations. Journal of Differential Equations, 31(1): 53-98, 1979.

[9] S. J. Fraser. The steady state and equilibrium approximations: A geometrical picture. The Journal of Chemical Physics, 88(8): 4732-4738, 1988.

[10] J.-M. Ginoux. Differential geometry applied to dynamical systems. World Scientific, 2009.

[11] J.-M. Ginoux and B. Rossetto. Differential geometry and mechanics: applications to chaotic dynamical systems. International Journal of Bifurcation and Chaos, 16(4): 887-910, 2006.

[12] J.-M. Ginoux, B. Rossetto, and L. O. Chua. Slow invariant manifolds as curvature of the flow of dynamical systems. International Journal of Bifurcation and Chaos, 18(11): 3409-3430, 2008.

[13] H. G. Kaper and T. J. Kaper. Asymptotic analysis of two reduction methods for systems of chemical reactions. Physica D: Nonlinear Phenomena, 165(1-2): 66-93, 2002.

[14] S. H. Lam and D. A. Goussis. The CSP method for simplifying kinetics. International Journal of Chemical Kinetics, 26: 461-486, 1994.

[15] U. Maas and S. B. Pope. Simplifying chemical kinetics: Intrinsic low-dimensional manifolds in composition space. Combustion and Flame, 88(3-4): 239-264, 1992.

[16] M. Okuda. A new method of nonlinear analysis for shaping and threshold actions. Journal of the Physical Society of Japan, 41(5): 1815-1816, 1976.

[17] E. Peacock-Lopez, D. B. Radov, and C. S. Flesner. Mixed-mode oscillations in a self-replicating dimerization mechanism. Biophysical Chemistry, 65(2-3): 171-178, 1997.

[18] B. Peng, V. Gaspar, and K. Showalter. False bifurcations in chemical systems: canards. Philosophical Transactions of the Royal Society of London. Series A: Physical and Engineering Sciences, 337(1646): 275-289, 1991.

[19] M. R. Roussel and S. J. Fraser. Geometry of the steady-state approximation: Perturbation and accelerated convergence methods. The Journal of Chemical Physics, 93(2): 1072-1081, 1990.

[20] A. N. Tikhonov. Systems of differential equations containing small parameters in the derivatives. Matematicheskii sbornik, 73(3): 575-586, 1952. 
[21] L. L. Tsai, G. R. Hutchison, and E. Peacock-López. Turing patterns in a self-replicating mechanism with a selfcomplementary template. The Journal of Chemical Physics, 113(5): 2003-2006, 2000.

[22] B. van der Pol. On relaxation-oscillations. Philosophical Magazine, 2(7): 978-992, 1927.

[23] A. Zagaris, C. W. Gear, T. J. Kaper, and Y. G. Kevrekidis. Analysis of the accuracy and convergence of equation-free projection to a slow manifold. ESAIM: Mathematical Modelling and Numerical Analysis, 43(4): 757-784, 2009.

[24] A. Zagaris, H. G. Kaper, and T. J. Kaper. Analysis of the computational singular perturbation reduction method for chemical kinetics. Journal of Nonlinear Science, 14: 59-91, 2004.

Université de la Rochelle, Laboratoire Mathématiques Images et Applications, EA3165, Avenue Michel Crépeau, 17042 la Rochelle Cedex, France

E-mail address: ebenoit@univ-lr.fr

Department of Applied Mathematics and Computer Science, Technical University of Denmark, 2800 KonGENS LyNGBY, DENMARK

E-mail address: mobr@dtu.dk

inRia Paris-Rocquencourt Research Centre, Project-Team MYCenae, Domaine de Voluceau, RocquenCourt BP 105, 78153 Le Chesnay Cedex, France

E-mail address: mathieu.desroches@inria.fr

inria Paris-Rocquencourt Research Centre, Project-Team MyCEnAe, Domaine de Voluceau, RocquenCOURT BP 105, 78153 Le Chesnay CEDEx, France

E-mail address: maciej.krupa@inria.fr 